\title{
Evaluation of melanin production by Sporothrix luriei
}

\author{
Ingrid Ludmilla Rodrigues Cruz, Maria Helena Galdino Figueiredo-Carvalho, Rosely Maria \\ Zancopé-Oliveira, Rodrigo Almeida-Paes/+
}

Fundação Oswaldo Cruz-Fiocruz, Instituto Nacional de Infectologia Evandro Chagas, Laboratório de Micologia, Rio de Janeiro, RJ, Brasil

There is a paucity of studies on the cell biology of Sporothrix luriei, the less common of the pathogenic Sporothrix species worldwide. The production of DHN-melanin, eumelanin, and pyomelanin were evaluated on the mycelial and yeast forms of the $S$. luriei ATCC 18616 strain. The mycelial form of this species produced only pyomelanin, which protected the fungus against environmental stressors such as ultraviolet light, heat, and cold. The yeast form was unable to produce any of the tested melanin types. The lack of melanin in the parasitic form of S. luriei may be an explanation for its low frequency in human infections.

Key words: Sporothrix luriei - L-tyrosine - pyomelanin

From 1898 to 2006, sporotrichosis was attributed to a single species Sporothrix schenckii (Kauffman 2006), or its variety, S. schenckii var. luriei (Padhye et al. 1992). With the advance of polyphasic fungal taxonomy, the single so-called $S$. schenckii species was separated into four species: $S$. schenckii sensu stricto, Sporothrix brasiliensis, Sporothrix globosa, and Sporothrix mexicana (Marimon et al. 2007). Moreover, S. schenckii var. luriei was elevated to the species level, and it is now called Sporothrix luriei (Marimon et al. 2008).

The first documented $S$. luriei infection occurred in 1956 (Ajello \& Kaplan 1969). Three other human sporotrichosis cases related to $S$. luriei have been reported (Mercadal-Peyrí et al. 1965, Alberici et al. 1989, Padhye et al. 1992). The main diagnostic feature in these cases was the presence of fungal eyeglasses-like cells (Padhye et al. 1992). A case in a dog, diagnosed through molecular methods, has also been reported (Oliveira et al. 2011).

Different from other Sporothrix species, the absence of sessile dark-pigmented conidia has been described for S. luriei (Marimon et al. 2008). Sporothrix pigmentation is the consequence of melanin deposition in the fungal cell wall (Almeida-Paes et al. 2017). Melanins are present in the three major pathogenic species of the genus: S. brasiliensis, S. schenckii, and S. globosa (AlmeidaPaes et al. 2015), and they protect these species against several stress conditions that they can face in the environment and during parasitism. Moreover, genomic data have revealed that melanin biosynthesis in S. schenckii and $S$. brasiliensis is similar (Almeida-Paes et al. 2017). To the best of our knowledge, there is no information about melanin in the $S$. mexicana cell wall. Since it was

doi: 10.1590/0074-02760170339

Financial support: CNPq (grant No. 449184/2014-5).

RA-P and RMZ-O are supported in part by CNPq (grant No. 305487/2015-

9 and 304976/2013-0, respectively).

+ Corresponding author: rodrigo.paes@ini.fiocruz.br

Received 21 August 2017

Accepted 25 September 2017 reported that $S$. mexicana produces dematiaceous conidia, as does $S$. schenckii and S. brasiliensis (Marimon et al. 2007), the black pigment observed in S. mexicana conidia is also thought to be related to melanin deposited in the cell wall of this species.

The lack of melanin in S. luriei is a possible hypothesis for its low prevalence in human infections. Therefore, this study aimed to determine whether this species can produce the three major types of fungal melanins (DHNmelanin, eumelanin, and pyomelanin) under well-established in vitro conditions used to study melanisation in other Sporothrix species.

The $S$. luriei strain INCQS 40253 (ATCC 18616 / CBS 937.72) was used in this study. The $S$. brasiliensis type strain (CBS 120339) was included as a control for melanin production. Strains were maintained in the mycelial form in Sabouraud dextrose agar at $25^{\circ} \mathrm{C}$ and in the yeast form in brain heart infusion agar at $35^{\circ} \mathrm{C}$. Production of DHN-melanin was assessed in a minimal medium (29.4 mM KH $\mathrm{PO}_{4}, 10 \mathrm{mM} \mathrm{MgSO}, 13 \mathrm{mM}$ glycine, $15 \mathrm{mM}$ dextrose, $3 \mu \mathrm{M}$ thiamine, $\mathrm{pH}$ 5.5). Experiments to detect eumelanin and pyomelanin were performed in minimal medium supplemented with $1 \mathrm{mM} \mathrm{L}$-dopa or $10 \mathrm{mM}$ L-tyrosine, respectively. Tricyclazole (16 mg/L), glyphosate $(100 \mathrm{mM})$, and sulcotrione $(16 \mathrm{mg} / \mathrm{L})$ were used to supplement the media to evaluate the blockage of the DHN-melanin, eumelanin, and pyomelanin metabolic pathways, respectively (Almeida-Paes et al. 2009, 2012, Teixeira et al. 2010).

Both the mycelial and yeast forms of $S$. luriei and the control $S$. brasiliensis strains were tested for melanin production at an initial inoculum concentration of $1 \times$ $10^{6}$ conidia or yeasts $/ \mathrm{mL}$ in the above described media. Fungi were incubated in the dark for 15 days at $25^{\circ} \mathrm{C}$ (conidia) or $35^{\circ} \mathrm{C}$ (yeasts) on a rotary incubator at 150 rpm. To detect DHN-melanin or eumelanin, cells were harvested from the cultures described above and washed three times in phosphate-buffered saline (PBS) and suspended in $1 \mathrm{M}$ sorbitol/0.1 $\mathrm{M}$ sodium citrate solution. Protoplasts were generated by incubating cells at $30^{\circ} \mathrm{C}$ in a solution containing $10 \mathrm{mg} / \mathrm{mL}$ cell wall-lysing enzymes (from Trichoderma harzianum; Sigma Chemi- 


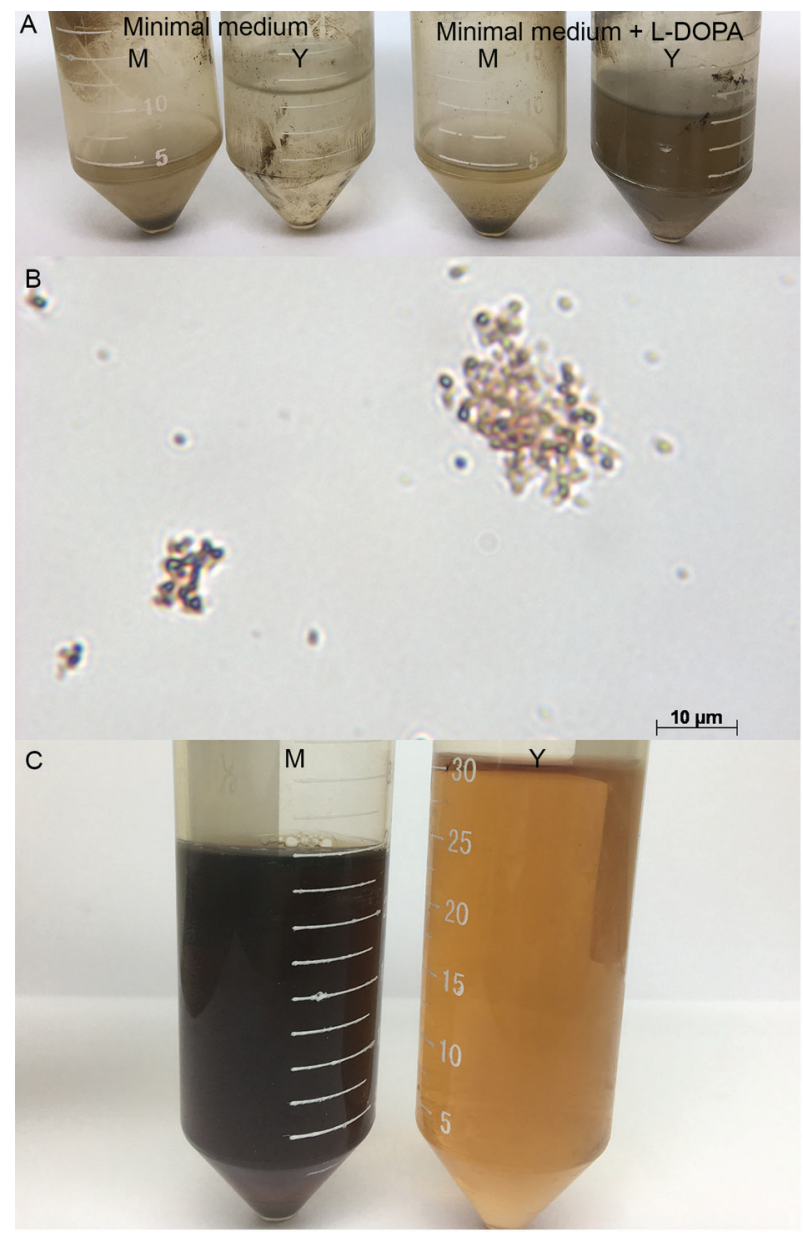

Fig. 1: evaluation of melanin production by Sporothrix luriei. (A): macroscopic aspects of hot-acid resistant particles yielded by mycelial (M) and yeast $(\mathrm{Y})$ cells grown in minimal medium and minimal medium supplemented with $1 \mathrm{mM}$ L-DOPA; (B): microscopic aspects of the hotacid resistant particles, bar: $10 \mu \mathrm{m}$; (C): black-soluble pigment observed in the supernatant of the mycelial (M) culture in minimal medium supplemented with $10 \mathrm{mM}$ L-tyrosine and absence of the pigment in the culture supernatant of the yeast culture in the same medium.

cal Co.) for $1 \mathrm{~h}$ at room temperature. Protoplasts were washed with PBS and incubated in 4.0 M guanidine thiocyanate for $1 \mathrm{~h}$ at room temperature with frequent vortexing. The resulting material was washed three times in PBS and boiled in $6.0 \mathrm{M}$ hydrochloric acid for $1 \mathrm{~h}$. Supernatants of cultures supplemented with L-tyrosine were filtered through $0.22-\mu \mathrm{M}$ membranes, acidified to $\mathrm{pH} 2.0$ using 0.5 $\mathrm{M}$ hydrochloric acid, and left for $24 \mathrm{~h}$ at room temperature. The precipitated pyomelanin was harvested by centrifugation $(12,800 \times g)$ and resuspended in sterile distilled water.

As expected, the control S. brasiliensis strain produced the three melanin types in both morphologies, as described previously (Supplementary data, Figure). In contrast, the chemical treatment with enzymes, denaturant, and hot acid dissolved S. luriei mycelial and yeast cells without generating dark particles retaining the shape and size of the conidia, hyphae, or yeast cells (Fig. 1A). However, small dystrophic particles, similar to those observed when the DHN-melanin synthesis was blocked by tricyclazole in S. brasiliensis or S. schenckii, were observed in both fungal morphologies, even in the absence of this inhibitor (Fig. 1B). The S. luriei yeast form was also unable to produce pyomelanin under the in vitro conditions employed herein. However, supernatants of S. luriei mycelial cultures supplemented with L-tyrosine turned black after 10 days of growth at $25^{\circ} \mathrm{C}$ (Fig. 1C). This pigment was acid resistant, and its synthesis was specifically blocked by sulcotrione, thereby confirming this pigment to be pyomelanin.

Since the $S$. luriei mycelial form produced pyomelanin, we hypothesised that this pigment would be involved in protection against harsh environmental conditions. To check this hypothesis, S. luriei conidia were harvested from cultures with and without L-tyrosine, adjusted to $1 \times 10^{8}$ conidia/mL, and submitted to either $15,30,45$, 60 , or 75 seconds of ultraviolet (UV) light $\left(290 \mu \mathrm{W} / \mathrm{cm}^{2}\right)$. In addition, conidia were incubated for $24 \mathrm{~h}$ at $38^{\circ} \mathrm{C}$ and stored without cryoprotectants at $4^{\circ} \mathrm{C}$ for six months to evaluate heat and cold protection, respectively. Six measurements were taken in each of these experiments. The results were analysed with the Mann-Whitney test using GraphPad 5 software. As depicted in Fig. 2A, melanised conidia submitted to UV light had more colony forming units than non-melanised conidia $(\mathrm{p}<0.05)$. Moreover, only melanised conidia survived UV exposures longer than 60 s. Melanised $S$. luriei conidia were also more resistant to heat and cold ( $\mathrm{p}<0.05$ for both experiments) than non-melanised cells (Fig. 2B).

The presence of pyomelanin in the mycelial form of $S$. luriei may be a result of the better adaptation of this species to environmental conditions, which agrees with the protection that this pigment confers to the fungus
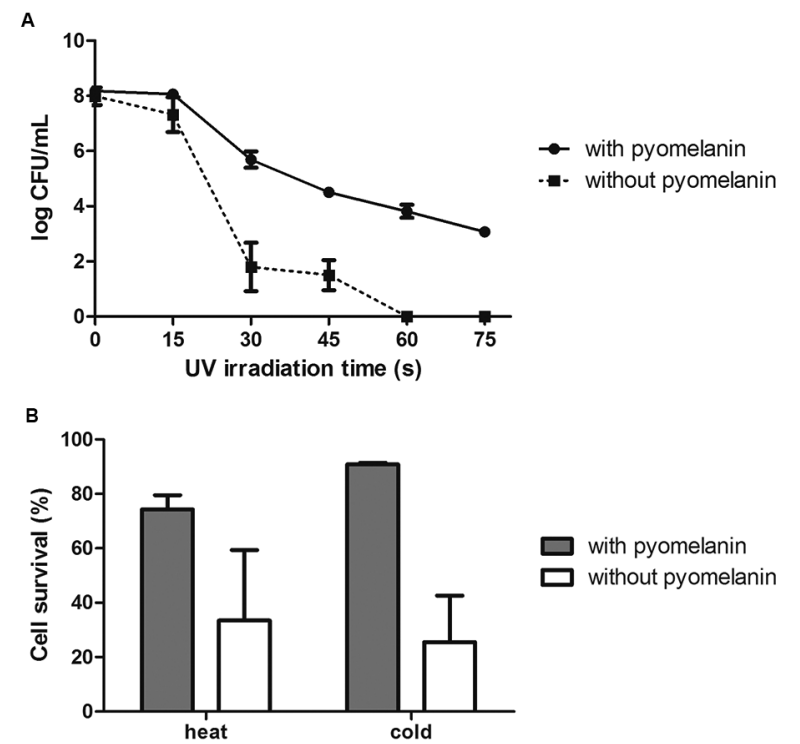

Fig. 2: resistance of melanised Sporothrix luriei conidia against environmental stressors. (A) growth $(\mathrm{CFU} / \mathrm{mL}$; mean \pm standard deviation) of cultures with and without pyomelanin after ultraviolet light irradiation for different exposure times; (B) percent survival (mean \pm standard deviation) of conidia with and without pyomelanin after heat $\left(38^{\circ} \mathrm{C}\right)$ and cold $\left(4^{\circ} \mathrm{C}\right)$ exposures. For all conditions, $\mathrm{p}<0.05$. 
against abiotic stress factors. The degree of protection against UV radiation observed in this study was similar to that observed with $S$. brasiliensis pyomelanin and other fungal melanin types (Almeida-Paes et al. 2012).

Melanins were not found in the $S$. luriei yeast cell wall. Its low incidence as an agent of sporotrichosis (Zhang et al. 2015) and the requirement of a high S. luriei inoculum to achieve virulence in an experimental infection model using the same strain as in the present study (Fernández-Silva et al. 2012) may result from the lack of melanin in the parasitic form of this species. Under the same conditions that other Sporothrix species are able to produce DHN- and eumelanin (AlmeidaPaes et al. 2009), only small acid-resistant particles that did not have the shape and size of $S$. luriei cells were observed. Besides the three melanin types studied in this work, some fungi produce other pigments, such as $\gamma$-glutaminyl-3,4-dihydroxy-benzene-melanin, catechol melanin, $p$-aminophenol melanin, deoxybostrycoidinmelanin, and asp-melanin. The observed particles are not likely to be related to these uncommon types of fungal melanins, since they are expressed in sexual reproduction structures and/or require exogenous compounds for production (Toledo et al. 2017). The black acid-resistant structures of $S$. luriei are similar to those produced by $S$. schenckii and S. brasiliensis when the DHN-pathway is inhibited with tricyclazole (Almeida-Paes et al. 2009), suggesting that melanin synthesis in $S$. luriei is blocked by an unknown mechanism. These dysmorphic particles resemble the melanosome-like structures observed in $S$. schenckii (Almeida-Paes et al. 2017). One hypothesis is that they are polymerisation products of accumulated intermediary metabolites of a hindered melanin synthesis pathway. Since information on the whole genome of $S$. luriei is unavailable, a search for mutations or missing genes related to melanin synthesis was not possible.

Due to the paucity of available $S$. luriei strains (Marimon et al. 2008), we were able to study melanisation in only one strain. This was also a limitation in other important studies on $S$. luriei taxonomy and virulence (Marimon et al. 2008, Oliveira et al. 2011, FernándezSilva et al. 2012). Future studies with more strains are necessary to gain a better understanding of $S$. luriei cell biology and pathogenesis.

\section{ACKNOWLEDGEMENTS}

To Marília Martins Nishikawa for providing the Sporothrix luriei strain used in this work.

\section{AUTHORS' CONTRIBUTION}

RA-P and RMZ-O conceived the study and wrote the manuscript; ILRC and MHGF-C performed the experiments; RA-P analysed the data. All authors read and approved the final manuscript.

\section{REFERENCES}

Ajello L, Kaplan W. A new variant of Sporothrix schenckii. Mykosen. 1969; 12(11): 633-44.

Alberici F, Paties CT, Lombardi G, Ajello L, Kaufman L, Chandler F. Sporothrix schenckii var luriei as the cause of sporotrichosis in Italy. Eur J Epidemiol. 1989; 5(2): 173-7.

Almeida-Paes R, Borba-Santos LP, Rozental S, Marco S, ZancopéOliveira RM, Cunha MML. Melanin biosynthesis in pathogenic species of Sporothrix. Fungal Biol Rev. 2017; 31(1): 50-9.

Almeida-Paes R, Frases S, Araujo GS, Oliveira MME, Gerfen GJ, Nosanchuk JD, et al. Biosynthesis and functions of a melanoid pigment produced by species of the Sporothrix complex in the presence of L-tyrosine. Appl Environ Microbiol. 2012; 78(24): 8623-30.

Almeida-Paes R, Frases S, Monteiro PCF, Gutiérrez-Galhardo MC, Zancopé-Oliveira RM, Nosanchuk JD. Growth conditions influence melanization of Brazilian clinical Sporothrix schenckii isolates. Microbes Infect. 2009; 11(5): 554-62.

Almeida-Paes R, Oliveira LC, Oliveira MME, Gutiérrez-Galhardo MC, Nosanchuk JD, Zancopé-Oliveira RM. Phenotypic characteristics associated with virulence of clinical isolates from the Sporothrix complex. BioMed Res Int. 2015; 2015: 212308.

Fernández-Silva F, Capilla J, Mayayo E, Guarro J. Virulence of Sporothrix luriei in a murine model of disseminated infection. Mycopathologia. 2012; 173(4): 245-9.

Kauffman CA. Endemic mycoses: blastomycosis, histoplasmosis, and sporotrichosis. Infect Dis Clin North Am. 2006; 20(3): 645-62.

Marimon R, Cano J, Gené J, Sutton DA, Kawasaki M, Guarro J. Sporothrix brasiliensis, S. globosa, and S. mexicana, three new Sporothrix species of clinical interest. J Clin Microbiol. 2007; 45(10): 3198-206.

Marimon R, Gené J, Cano J, Guarro J. Sporothrix luriei: a rare fungus from clinical origin. Med Mycol. 2008; 46(6): 621-5.

Mercadal-Peyrí J, Bassas-Grau M, Sans-Macaró J, de Martín-Gassó C, Mercadal-Peyrí JO. Two very rare clinical diseases in our climate: cutaneous actinomycoses and blastomycosis of a vegetating type. Mycopathol Mycol Appl. 1965; 27(1): 68-74.

Oliveira DC, Lopes PGM, Spader TB, Mahl CD, Tronco-Alves GR, Lara VM, et al. Antifungal susceptibilities of Sporothrix albicans, S. brasiliensis, and S. luriei of the S. schenckii complex identified in Brazil. J Clin Microbiol. 2011; 49(8): 3047-9.

Padhye AA, Kaufman L, Durry E, Banerjee CK, Jindal SK, Talwar $\mathrm{P}$, et al. Fatal pulmonary sporotrichosis caused by Sporothrix schenckii var. luriei in India. J Clin Microbiol. 1992; 30(9): 2492-4.

Teixeira PAC, de Castro RA, Ferreira FRL, Cunha MML, Torres AP, Penha CVLY, et al. L-DOPA accessibility in culture medium increases melanin expression and virulence of Sporothrix schenckii yeast cells. Med Mycol. 2010; 48(5): 687-95.

Toledo AV, Franco MEE, Lopez SMY, Troncozo MI, Saparrat MCN, Balatti PA. Melanins in fungi: types, localization and putative biological roles. Physiol Mol Plant Pathol. 2017; 99: 2-6.

Zhang Y, Hagen F, Stielow B, Rodrigues AM, Samerpitak K, Zhou $\mathrm{X}$, et al. Phylogeography and evolutionary patterns in Sporothrix spanning more than 14000 human and animal case reports. Persoonia. 2015 ; 35: 1-20. 